\title{
Fire patterns in the Brazilian Cerrado: an approach comparing different input datasets in the fire risk modelling
}

\author{
Guilherme Martins $^{1 *}$, Joana Nogueira ${ }^{l}$, Alberto Setzer ${ }^{l}$, Fabiano Morelli $^{l}$
}

\footnotetext{
${ }^{1}$ National Institute for Space Research, São José dos Campos, Brazil

*e-mail: guilherme.martins@inpe.br
}

\begin{abstract}
Emissions from vegetation fires are relevant in the atmosphere-biosphere interaction. Nevertheless, fire is still intensely used as a tool in land management, modifying natural fire patterns in fire-prone ecosystems. The Brazilian Cerrado has shown increased anthropogenic fire ignitions, especially due to deforestation that removed $\sim 50 \%$ of its original cover and unusual droughts. Fire risk (FR) models using meteorological and vegetation parameters have been used to estimate fire patterns at biome level. The aim of this study was to evaluate the performance of INPE's FR model using different climate and land cover (LC) datasets (versions 0 and 1) to estimate FR patterns in the Cerrado. Meteorological datasets from CoSch and MCD12Q1-IGBP V006 land cover data represent v0 while v1 is composed by IMERG and Mapbiomas v3.0 datasets. The analyses were performed in the wet (W: November-March) and dry (D: May-September) seasons from 2015 to 2018 at $1 \mathrm{~km}$ of spatial resolution. The versions were compared using the seasonal predominance of FR (PFR) and evaluated in five categories: "minimum", $\mathrm{FR}<=0.15$; "low", $0.15<\mathrm{FR}<=0.40$; "medium", $0.40<\mathrm{FR}<=0.70$; "high", $0.70<\mathrm{FR}<=0.95$ and "critical", $0.95<\mathrm{FR}<=1.0$. The main fire pattern differences between $\mathrm{v} 0$ and v1 were observed in D, when the PFR remains "high" during all season according to v0, while v1classifies "critical" PFR from July to September. In W, differences were not observed, except for November, classified as "low" PFR by v0 and "minimum" PFR in v1. These differences can be related to the higher LC spatial resolution and definition of vegetation types in v1 such as woody savannas; $v 1$ is based on Landsat medium resolution spectral images $(\sim 30 \mathrm{~m})$ while v0 uses MODIS low resolution $(\sim 500 \mathrm{~m})$. Concerning precipitation, the information has a higher spatial consistency using $10 \mathrm{~km}$ of spatial resolution in $\mathrm{v} 1$ while v0 uses $25 \mathrm{~km}$ of spatial resolution. With new Mapbiomas editions and revisions released every year, INPE's FR will be updated accordingly, allowing a realistic temporal modeling of the vegetation; including terrain data in this condition will allow a new FR product at $30 \mathrm{~m}$ resolution for protected areas - our next goal.
\end{abstract}

Keywords: Fire modelling; fire season; savannas; Brazil; land use 